TITLE:

\title{
High-quality antiferromagnetic EuTiO3 epitaxial thin films on SrTiO3 prepared by pulsed laser deposition and postannealing
}

\section{$\operatorname{AUTHOR}(\mathrm{S}):$}

Fujita, Koji; Wakasugi, Naoki; Murai, Shunsuke; Zong, Yanhua; Tanaka, Katsuhisa

\section{CITATION:}

Fujita, Koji ... [et al]. High-quality antiferromagnetic EuTiO3 epitaxial thin films on SrTiO3 prepared by pulsed laser deposition and postannealing. APPLIED PHYSICS LETTERS 2009, 94(6): 062512.

\section{ISSUE DATE:}

2009-02

URL:

http://hdl.handle.net/2433/109898

\section{RIGHT:}

Copyright 2009 American Institute of Physics. This article may be downloaded for personal use only. Any other use requires prior permission of the author and the American Institute of Physics. The following article appeared in APPLIED PHYSICS LETTERS 94, 062512 (2009) and may be found at http://link.aip.org/link/APPLAB/v94/i6/p062512/s1 


\title{
High-quality antiferromagnetic $\mathrm{EuTiO}_{3}$ epitaxial thin films on $\mathrm{SrTiO}_{3}$ prepared by pulsed laser deposition and postannealing
}

\author{
Koji Fujita, ${ }^{\text {a) }}$ Naoki Wakasugi, Shunsuke Murai, Yanhua Zong, and Katsuhisa Tanaka \\ Department of Material Chemistry, Graduate School of Engineering, Kyoto University, \\ Katsura, Nishikyo-ku, Kyoto 615-8510, Japan
}

(Received 26 September 2008; accepted 22 December 2008; published online 13 February 2009)

\begin{abstract}
We report on epitaxial growth and magnetic properties of $\mathrm{EuTiO}_{3}$ thin films with a perovskite structure. Single crystalline $\mathrm{EuTiO}_{3}$ films with atomically flat surface are grown on (001) surface of $\mathrm{SrTiO}_{3}$ by pulsed laser deposition (PLD) and subsequent annealing in reducing atmosphere. The as-deposited films possess the crystal structure with an elongated $c$-axis and tend to stabilize ferromagnetically ordered $\mathrm{Eu}^{2+}$ spins at low temperatures. Postannealing at $1000{ }^{\circ} \mathrm{C}$ relaxes the out-of-plane lattice strain, and brings about a drastic change in magnetic structure; the annealed film becomes an antiferromagnet below Néel temperature of $5.1 \mathrm{~K}$. The change in magnetic properties accompanied by the modification in lattice constant is discussed. (C) 2009 American Institute of Physics. [DOI: 10.1063/1.3072598]
\end{abstract}

An interest in multiferroics, where ferroelectric and ferromagnetic (FM) or antiferromagnetic (AFM) orderings coexist, has been recently revived because of their potential applications to multifunctional magnetoelectric and magneto-optical devices. ${ }^{1,2}$ In particular, the discovery of new multiferroics such as $\mathrm{TbMnO}_{3},{ }^{3} \mathrm{BiFeO}_{3},{ }^{4}$ and $\mathrm{BiMnO}_{3}{ }^{5}$ triggered rapid developments in the exploration of materials with a strong dielectric-magnetic coupling so that a small modulation in magnetic (electric) field can induce a large change in electric polarization (magnetization). One strategy to achieve the strong dielectric-magnetic coupling is to select a system where magnetization couples to a specific infraredallowed optical phonon mode with low frequency, i.e., a soft phonon mode. In such a case, a gigantic change in dielectric constant is expected to occur by an ordering of magnetic moments, since the energy scale of soft mode is comparable to that of magnetic interaction.

$\mathrm{EuTiO}_{3}$ is a unique material having a strong coupling between magnetic ordering and soft phonon mode. Bulk $\mathrm{EuTiO}_{3}$ adopts a cubic perovskite structure $\left(\mathrm{Eu}^{2+} \mathrm{Ti}^{4+} \mathrm{O}_{3}\right.$, space group $P m \overline{3} m$ ) at room temperature. ${ }^{6,7}$ The absence of electrons in Ti $3 d$ orbital means that $\mathrm{EuTiO}_{3}$ is a band insulator. Large magnetic moments localized on Eu sites $(J=S$ $=7 / 2)$ order below the Néel temperature $\left(T_{\mathrm{N}}=5.5 \mathrm{~K}\right)$ to form a $G$-type antiferromagnet. Interestingly, the dielectric constant varies in response to the ordering states of Eu spins due to the coupling between the localized spins and a soft phonon mode. ${ }^{8}$

Another important aspect of the coupling between phonon and magnetic moments appears when a soft phonon mode is sensitive to lattice strains. This effect can be the most prominent in the form of thin film; an epitaxial strain due to the lattice mismatch between film and substrate has a great impact on the dielectric and magnetic properties. Recently, Fennie and Rabe ${ }^{9}$ calculated the dielectric properties of $\mathrm{EuTiO}_{3}$ in $\mathrm{FM}$ or AFM phases under biaxial compressive strain and showed that the most stable magnetic phase can be tuned by experimentally attainable lattice strain. A similar

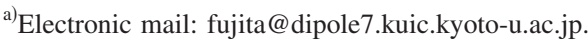

attempt was performed by Ranjan et al. ${ }^{10}$ to calculate the stability of several magnetic phases as a function of lattice volume.

In spite of such interesting theoretical predictions and of practical importance in making devices, the fabrication of $\mathrm{EuTiO}_{3}$ epitaxial thin film has been rarely reported. ${ }^{11} \mathrm{Re}-$ cently, we have fabricated the thin films of $\mathrm{EuTiO}_{3}$ on $\mathrm{SrTiO}_{3}(001)$ substrates by PLD under the different deposition conditions from those in Ref. 11 and reported their magnetic properties. ${ }^{12}$ Although the as-deposited films could be obtained as a single phase of $\mathrm{EuTiO}_{3}$, the out-of-plane lattice constant was larger (typically $\sim 2.6 \%$ ) than the bulk value, and an FM-like behavior was observed at low temperatures, in contrast to the AFM behavior of bulk $\mathrm{EuTiO}_{3}$.

In this study, we report the fabrication of single crystalline and $\mathrm{AFM} \mathrm{EuTiO}$ thin films by postannealing of the as-deposited film in reducing atmosphere. According to the structural analysis utilizing conversion electron Mössbauer spectroscopy (CEMS) and x-ray diffraction (XRD), the postannealing relaxes the out-of-plane lattice expansion to produce $\mathrm{AFM} \mathrm{EuTiO}_{3}$. The variation in magnetic behavior by postannealing is explainable in terms of the change in lattice constant, which is in good agreement with recent theoretical studies. ${ }^{9,10}$

$\mathrm{EuTiO}_{3}$ thin films were grown on atomically flat $\mathrm{SrTiO}_{3}$ (001) substrates (Shinkosha Co. Ltd.) by PLD. A KrF excimer laser $(248 \mathrm{~nm}, 5 \mathrm{~Hz})$ was focused on a sintered $\mathrm{Eu}_{2} \mathrm{Ti}_{2} \mathrm{O}_{7}$ ceramic target at a fluence of $2 \mathrm{~J} / \mathrm{cm}^{2}$. The substrate temperature was maintained at $650{ }^{\circ} \mathrm{C}$ and the oxygen pressure was $1.0 \times 10^{-5} \mathrm{~Pa}$. After the deposition, the film was annealed at $1000{ }^{\circ} \mathrm{C}$ under a flowing gas of 95 vol \% Ar +5 vol $\% \mathrm{H}_{2}$ for $8.5 \mathrm{~h}$ to prevent the oxidation of $\mathrm{Eu}^{2+}$. The thickness and chemical composition of films were characterized by the Rutherford backscattering using a $2.0 \mathrm{MeV} \mathrm{He}^{2+}$. The analysis of the annealed film revealed a stoichiometric cation ratio (1:1) of Eu and Ti. The film thickness was evaluated to be about $200 \mathrm{~nm}$.

The crystal structure of the films was estimated by highresolution XRD measurements using $\mathrm{Cu} K \alpha$ radiation. The $2 \theta-\omega$ XRD patterns for as-deposited and annealed films are shown in Fig. 1(a). For the as-deposited film, sharp Bragg 

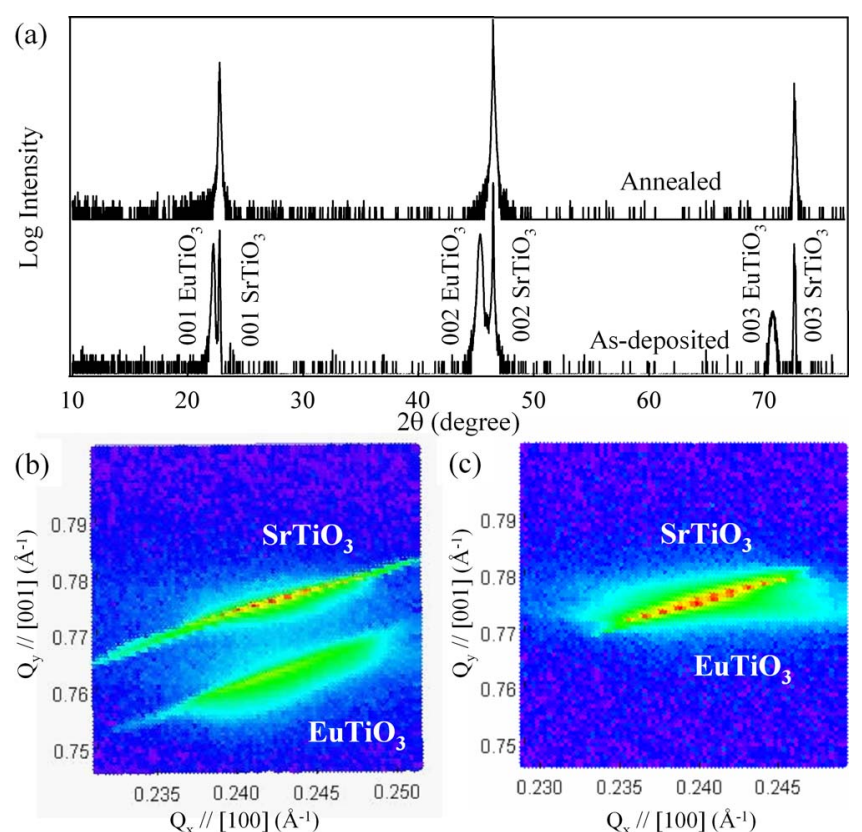

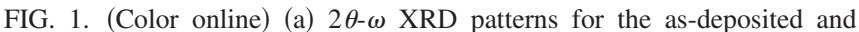
annealed $\mathrm{EuTiO}_{3}$ thin films. RSMs at around 301 diffraction are displayed for the (b) as-deposited and (c) annealed thin films.

peaks for $\mathrm{EuTiO}_{3} 00 n(n=1,2$, and 3) are observed together with $\mathrm{SrTiO}_{3}$ 00n peaks, indicating the epitaxial growth of $\mathrm{EuTiO}_{3}$ oriented along the $c$-axis. The out-of-plane lattice constant calculated from the 003 peak is $0.3993 \mathrm{~nm}$, which is much larger than the reported bulk value of $0.3905 \mathrm{~nm}^{8}$ Upon postannealing, $\mathrm{EuTiO}_{3}$ peaks shift toward higher angle side and are superimposed on the $\mathrm{SrTiO}_{3}$ peaks without yielding any impurity phases. Namely, the crystal lattice of the as-deposited film shrinks along the $c$-axis by postannealing so that the out-of-plane lattice constant $(0.3900 \mathrm{~nm})$ is almost the same as the bulk value.

To fully examine the strain states, $\mathrm{x}$-ray reciprocal space mapping (RSM) was measured for the 301 diffraction using a four-circle XRD apparatus. Figures 1(b) and 1(c) show the RSMs for the as-deposited and annealed films, respectively. Two Bragg spots from film and substrate are observed for the as-deposited film. These two spots have the same $Q_{x}$, while $Q_{y}$ of $\mathrm{EuTiO}_{3}$ is smaller than that of $\mathrm{SrTiO}_{3}$. This result indicates that the crystal lattice of the as-deposited film elongates unidirectionally along the $c$-axis, as implied by $2 \theta-\omega$ scan. For the annealed film, in contrast, $Q_{x}$ and $Q_{y}$ of $\mathrm{EuTiO}_{3}$ coincide well with those of $\mathrm{SrTiO}_{3}$, meaning that the crystal structure of $\mathrm{EuTiO}_{3}$ is relaxed by postannealing.

Surface morphology of the films was observed by an atomic force microscope. A stepped and terraced structure is clearly seen for the annealed film [Fig. 2(a)]; the step height is approximately $0.4 \mathrm{~nm}$ [Fig. 2(b)], corresponding to the size of one unit cell of bulk $\mathrm{EuTiO}_{3}$. The as-deposited film exhibited the characteristic of two-dimensional growth (not shown), reflecting the atomically flat surface of substrates. We believe that the postannealing of the as-deposited films on nearly perfectly lattice-matched $\mathrm{SrTiO}_{3}$ substrates serves to reduce the lattice constant of $c$-axis without yielding defects such as grain boundaries and pits, leading to the single crystalline films with atomically flat and smooth surfaces.

Valence states of europium ions in the as-deposited and annealed films were characterized by room-temperature ${ }^{151}$ Eu CEMS spectra, as shown in Fig. 3(a), using ${ }^{151} \mathrm{Sm}_{2} \mathrm{O}_{3}$
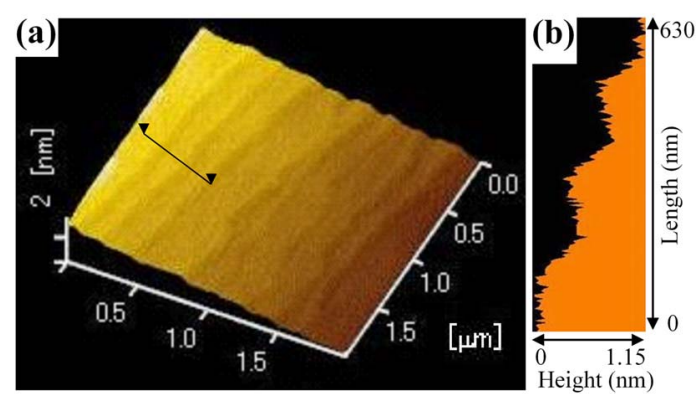

FIG. 2. (Color online) (a) Atomic force microscopy image $\left(2 \times 2 \mu \mathrm{m}^{2}\right)$ for the annealed $\mathrm{EuTiO}_{3}$ thin film grown on $\mathrm{SrTiO}_{3}$ (001) substrate. (b) Crosssectional profile of the image along the solid line indicated in (a)

as a $\gamma$-ray source. Calibration of Doppler velocity was made by using a spectrum of $\alpha$-Fe, which was also used as a standard for isomer shift. It is seen that the spectra are mainly composed of absorption due to $\mathrm{Eu}^{2+}$ at around $-13 \mathrm{~mm} \mathrm{~s}^{-1}$; the fractions of the absorption area of $\mathrm{Eu}^{2+}$ are estimated to be 0.96 and 0.98 for the as-deposited and annealed films, respectively. The remaining $\mathrm{Eu}^{3+}$ ions in the as-deposited film are slightly reduced to $\mathrm{Eu}^{2+}$ ions by postannealing. The impurity $\mathrm{Eu}^{3+}$ ions are probably accompanied by the formation of $\mathrm{Ti}^{3+}$ or interstitial $\mathrm{O}^{2-}$ to compensate for the excess positive charge. In Fig. 3(b), the magnetization $(M)$ of the as-deposited and annealed films is plotted as a function of temperature $(T)$. Field cooling (FC) and zero field cooling (ZFC) were performed using a superconducting quantum interference device magnetometer, while a dc magnetic field $\left(H_{\mathrm{dc}}\right)$ of 100 Oe was applied parallel to the film surface. One can see a large difference in magnetism between these two films; $M$ of the as-deposited film increases monotonically with decreasing $T$, while the annealed film exhibits a distinct AFM transition at $5.1 \mathrm{~K}$, in reasonable agreement with the value reported for bulk single-crystalline $\mathrm{EuTiO}_{3}{ }^{6,7}$ No divergence between $\mathrm{ZFC}$ and $\mathrm{FC}$ conditions was observed for both the as-deposited and annealed films.

Figure 4 compares the magnetic properties between the as-deposited and annealed films in more detail. Figure 4(a) depicted the $M-T$ curves at varied $H_{\mathrm{dc}}$ for the annealed film. The AFM transition is evident when $H_{\mathrm{dc}}$ is low like 100 and $1000 \mathrm{Oe}$, whereas the FM behavior is observed when $H_{\mathrm{dc}}$ $=20 \mathrm{kOe}$. In other words, the stable spin structure in the annealed film is converted from AFM to FM as $H_{\mathrm{dc}}$ applied

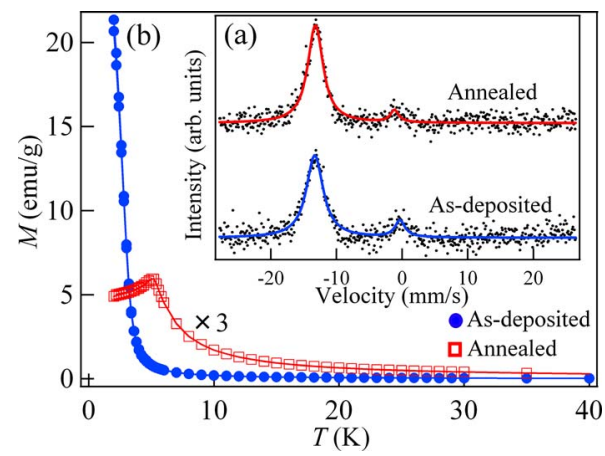

FIG. 3. (Color online) (a) ${ }^{151}$ Eu CEMS spectra for the as-deposited and annealed $\mathrm{EuTiO}_{3}$ thin films (dots). The solid lines represent the least-squares fits using two Lorentzians. (b) $M-T$ curves for the as-deposited (closed circles) and annealed (open squares) $\mathrm{EuTiO}_{3}$ thin films under $H_{\mathrm{dc}}$ $=100$ Oe applied along the $\langle 100\rangle$ direction. For both films, the ZFC and FC data fall on the same curve. The $M$ of the annealed film is multiplied by a factor of 3 for clarity. 
(a) Annealed

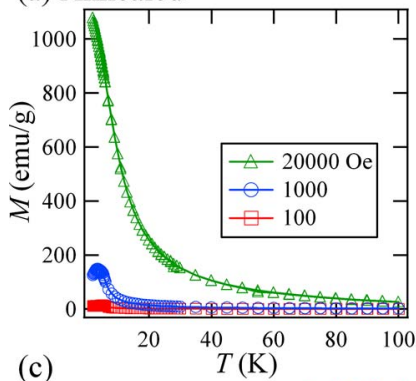

(c)

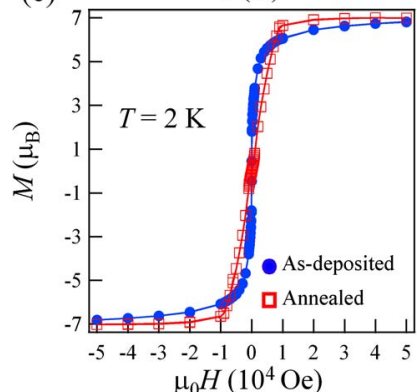

(b) As-deposited

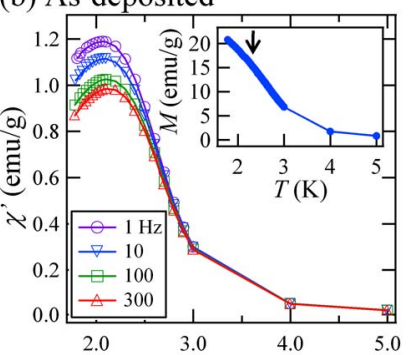

(d)

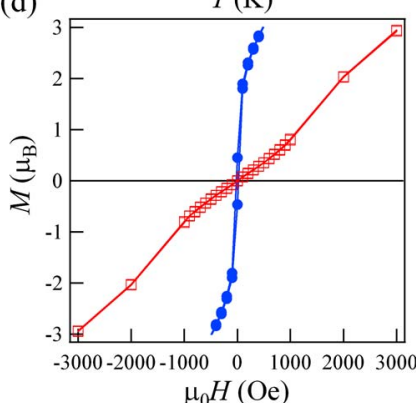

FIG. 4. (Color online) (a) $M-T$ curves for annealed $\mathrm{EuTiO}_{3}$ thin film at $H_{\mathrm{dc}}=100$ (open squares), 1000 (open circles), and 20000 Oe (open triangles) applied along the $\langle 100\rangle$ direction. (b) $\chi^{\prime}-T$ curves for the asdeposited $\mathrm{EuTiO}_{3}$ thin film at $H_{\mathrm{ac}}=3$ Oe and $H_{\mathrm{dc}}=0$ Oe. The ac frequencies are $1,10,100$, and $300 \mathrm{~Hz}$. The inset represents the low-temperature data of the $M-T$ curve for as-deposited film as shown in Fig. 3(b). The arrow denotes an inflection point. (c) Magnetic field $\left(\mu_{0} H\right)$ dependence of $M$ at $2 \mathrm{~K}$ for the as-deposited (closed circles) and annealed (open squares) $\mathrm{EuTiO}_{3}$ thin films. (d) Enlarged view of (c).

to the film is raised. This spin flopping behavior is quantitatively coincident with that of the bulk single-crystalline $\mathrm{EuTiO}_{3} .{ }^{8}$ On the other hand, a close inspection of the $M-T$ curve for the as-deposited film, as shown in Fig. 3(b), demonstrates an inflection point at around $2 \mathrm{~K}$ [inset in Fig. 4(b)], implying the presence of FM-like phase transition. To address the low-temperature magnetic behavior of the asdeposited film, ac susceptibility was measured under the ZFC condition. Figure 4(b) displays the dependence of the real part of ac susceptibility $\left(\chi^{\prime}\right)$ on $T$ at an ac magnetic field $\left(H_{\mathrm{ac}}\right)$ of 3 Oe in the absence of $H_{\mathrm{dc}}$. The $\chi^{\prime}-T$ curve exhibits a peak at around $2.2 \mathrm{~K}$, which depends only weakly on the ac frequency in the range of $1-300 \mathrm{~Hz}$. This result is indicative of the presence of the finite but long-range magnetic order in the as-deposited film; dynamical scaling analysis according to the conventional critical slowing down ${ }^{13}$ reveals the microscopic relaxation time $\tau_{0}=10^{-22} \mathrm{~s}$, far below the typical value for glassy magnetic systems $\left(\sim 10^{-13} \mathrm{~s}\right.$ for atomic spins and $>10^{-13} \mathrm{~s}$ for assemblages of coherent atomic spins). Figure 4(c) shows the magnetic field $\left(\mu_{0} H\right)$ dependence of $M$ at $2 \mathrm{~K}$ for the as-deposited and annealed films. The data at low $\mu_{0} H$ are magnified in Fig. 4(d). An abrupt increase in slope at around 1000 Oe for the annealed film corresponds to the spin flopping of AFM to FM alignment of Eu spins, while a steep slope at low $\mu_{0} H$ for the as-deposited film reflects an inherent FM alignment of Eu spins. For both films, the saturation magnetizations are around $7 \mu_{B}$ per one $\mathrm{Eu}$ ion, coincident with the theoretical spin-only magnetic moment of $\mathrm{Eu}^{2+}$, i.e., $J=S=7 / 2$.

As shown in Fig. 4, the as-deposited film tends to prefer FM alignment of Eu spins at low temperatures unlike the

annealed thin film. As ascertained by the analysis of XRD, whereas the annealed film possesses a lattice constant similar to the value of bulk $\mathrm{EuTiO}_{3}$, the out-of-plane lattice constant of the as-deposited film is longer than that of the bulk value. Fennie and Rabe $^{9}$ carried out the first principles densityfunctional calculations based on the generalized gradient approximation $(\mathrm{GGA})+U(U$ is the on-site Coulomb interaction in the Hubbard model) for both FM and $G$-type AFM spin arrangements of $\mathrm{EuTiO}_{3}$ under biaxial compressive strain in the $a b$-plane of the perovskite structure. They concluded that the $G$-type AFM phase is stable without the strain but that the FM phase becomes more stable when the strain exceeds $1.2 \%$. In their calculations the lattice volume was kept constant so that the compressive strain in the $a b$-plane corresponds to the tensile strain in the $c$-axis. Ranjan et al. ${ }^{10}$ performed similar calculations and found that the groundstate magnetic structure changes from $G$-type AFM to FM with an increase in the lattice volume when $U=6 \mathrm{eV}$. Our experimental results indicate that the elongation of the $c$-axis is apt to stabilize the FM alignment of Eu spins, coincident with the theoretical calculations. The elongation of the $c$-axis in the as-deposited $\mathrm{EuTiO}_{3}$ film is about $2.4 \%$ as evaluated from the variation in lattice constant with annealing. This value corresponds to the strain in the $a b$-plane of $1.2 \%$, which falls in the FM regime. ${ }^{9}$

In conclusion, single-crystalline $\mathrm{EuTiO}_{3}$ thin films with atomically flat surface have been fabricated on the $\mathrm{SrTiO}_{3}$ substrates by PLD and postannealing in reducing atmosphere. The annealed film possesses the lattice constant and $T_{\mathrm{N}}$ similar to the bulk $\mathrm{EuTiO}_{3}$. Also, we have obtained experimental evidence which supports the theoretical predictions ${ }^{9,10}$ that the magnetic interaction between nearestneighboring $\mathrm{Eu}^{2+}$ ions is converted from $\mathrm{AFM}$ to $\mathrm{FM}$ when the $c$-axis of $\mathrm{EuTiO}_{3}$ is increased.

The authors thank Y. Isozumi and M. Tosaki of Radioisotope Research Center, Kyoto University for the Mössbauer effect measurements. This research was financially supported by the Grant-in-Aid for Scientific Research from MEXT, Japan.

${ }^{1}$ S. W. Cheong and M. Mostovoy, Nature Mater. 6, 13 (2007).

${ }^{2}$ Y. Tokura, Science 312, 1481 (2006).

${ }^{3}$ T. Kimura, T. Goto, H. Shintani, K. Ishizaka, T. Arima, and Y. Tokura, Nature (London) 426, 55 (2003).

${ }^{4}$ J. Wang, J. B. Neaton, H. Zheng, V. Nagarajan, S. B. Ogale, B. Liu, D. Viehland, V. Vaithyanathan, D. G. Schlom, U. V. Waghmare, N. A. Spaldin, K. M. Rabe, M. Wuttig, and R. Ramesh, Science 299, 1719 (2003).

${ }^{5}$ T. Kimura, S. Kawamoto, I. Yamada, M. Azuma, M. Takano, and Y. Tokura, Phys. Rev. B 67, 180401(R) (2003).

${ }^{6}$ T. R. McGuire, M. W. Shafer, R. J. Joenk, H. A. Alperin, and S. J. Pickart, J. Appl. Phys. 37, 981 (1966).

${ }^{7}$ C.-L. Chien, S. DeBenedetti, and F. De S. Barros, Phys. Rev. B 10, 3913 (1974).

${ }^{8}$ T. Katsufuji and H. Takagi, Phys. Rev. B 64, 054415 (2001).

${ }^{9}$ C. J. Fennie and K. M. Rabe, Phys. Rev. Lett. 97, 267602 (2006).

${ }^{10}$ R. Ranjan, H. S. Nabi, and R. Pentcheva, J. Phys.: Condens. Matter 19, 406217 (2007).

${ }^{11}$ H.-H. Wang, A. Fleet, J. D. Brock, D. Dale, and Y. Suzuki, J. Appl. Phys. 96, 5324 (2004).

${ }^{12}$ K. Kugimiya, K. Fujita, K. Tanaka, and K. Hirao, J. Magn. Magn. Mater. 310, 2268 (2007).

${ }^{13}$ A. T. Ogielski, Phys. Rev. B 32, 7384 (1985). 